magnetised the Moon can ever be identified. As far as the lunar magnetisation process is concerned, Runcorn and Urey rejected TRM because the required sequence of temperature changes is difficult to envisage and rejected viscous magnetisation because of its fundamental instability. Instead, they favoured depositional magnetisation involving magnetised particles scttling nonturbulently through the accreting gas sphere in the external magnetic field, a process consistent with the existence of low temperatures. In this issue of Nature, Strangway and Sharpe now investigate a fourth possibility-isothermal magnetism acquired in a steady external field--in the light of the Moon's thermal evolution.

Peter J. Smith

\section{Dimensions of the possible}

\section{David Davies}

THE "Recognition of Alien Life", a symposium organised by N. W. Pirie (Rothamsted Experimental Station) at the Royal Society on May 2, could as well have been called the Carl Sagan Symposium. Sagan - from Cornell University-jetted in to London, talked to the press, gave the first lecture, was never far from other speakers' minds for the rest of the day, interjected wittily, moved on to lecture elsewhere and jetted out. And with good cause was he at the centre, for nobody can have thought more than he about life elsewhere.

Alien life may be intelligent or not; Sagan considered the intelligent sort. At present, he said, nothing is known about extraterrestrial intelligence, but the technology is now available for the analysis of signals from stars and the scanning of planetary surfaces. Critical experiments, in his view, are coming up soon. The best data with which to start are observations of the Earth such as, say, Martians could make (not that Sagan has much time for Martian intelligence). All depends on resolution, as a collection of satellite photographs show. At $1 \mathrm{~km}$ resolution he could find no sign of life on Earth-except at Cochran, Ontario! True, cities appear as dirty smudges, but they show no sign of reworking. A useful guide is 'departure from thermodynamic equilibrium' such as is shown by geometrisation of Earth features. At $100 \mathrm{~m}$ resolution, this begins to show up in, for instance, large field patterns. The nocturnal light of cities is obvious, but an observer would have doubts, as many large cities are on waterfronts; maybe some chemical process could generate the light.

Eavesdroppers at stellar distances on radio emissions from Earth would be struck by the enormous energy density around metre wavelengths from radio, radar and television. These signals would be detectable at tens of light years and intelligent terrestrial life would rapidly be inferred from their highly ordered nature. With radio telescopes the range could be a thousand times greater, though given limited facilities, it is better, Sagan belicves, to receive than transmit as man is likely to be the backward partner. The art of anticryptography-devising self evident messages for other intelligent life-is growing.

C. H. Waddington (University of Edinburgh) also considered intelligent alien life in trying to pin down what tells human beings that an artefact is just that. Unless one stumbles across a mechanical device, it is probable that repetition of shapes is as good a guide as any to intelligence. P. H. Gregory (Rothamsted) stressed that in talking about the recognition of life at the microscopic level the decision is largely an aesthetic one; on Earth man is still inadequately aware of the realms of possibility. He proposed an atlas of terrestrial microscopic objects as a valuable step towards improving methodology.

At this stage the meeting became philosophical as the discussion circled inconclusively round the question from the floor, "What is life?", reminding me of Gluck, my neighbour of Shelley and Sir Peter Medawar of flogging a dead horse.

The next contribution, by J. E. Lovelock (University of Reading), was in many ways the most fascinating. What could astronomical observations reveal about the probable existence of a biosphere? Thermodynamic disequilibrium in the atmosphere could be a good guide to the existence of life, and on Earth gross departures from equilibrium in nitrogen, hydrogen and methane point to life being present, a conclusion which multiplex spectrometry does not endorse for other planets.

The remainder of the meeting was devoted to questions of life on Earth, its past record, its energy relationships and its evolution. I. R. Kaplan (University of California, Los Angeles) reviewed stable isotope analysis, particularly of hydrogen, carbon, oxygen, nitrogen and sulphur. Variations in concentration ratios by biological enrichment are helpful in understanding past terrestrial environments. This scheme was taken further by $P$. C. Sylvester-Bradley (University of Leicester) for the Precambrian geological record. The origin of life is placed at about 4,000 million years ago (a review of this field appeared in Nature, 248, 730; 1974). J. De Ley (State University, Gent) discussed energy sources and storage and remarked on the adenine-based nature of life on Earth through the dominance of ATP as an energy carrier. Finally, A. G. Cairns-Smith (University of Glasgow) speculated on the role of clays as possible catalysts or frameworks for the evolution of biochemicals from inorganic origins.

Medawar summed up characteristically: "The heterogeneity of life and the varieties of metabolism and modes of behaviour are so enormous that nothing should surprise us about alien life. All we can do is attempt to sketch out the dimensions of the possible".

\section{Alloy glasses and beyond}

from a Correspondent

A $\mathrm{T}$ the eighth International Thin Films Conference held at the University of Sussex from April 8 to 10 under the sponsorship of the Thin Films and Surfaces group of the Institute of Physics, the most noticeable feature was the diversity of topics discussed: many of the phenomena of solid state physics have now been reproduced in thin film form, and for good measure some novel phenomena have been discovered.

The growth in the technology of electron-optical instruments continues to provide new and valuable aids to the structural investigation of thin films. According to M. Prutton (University of York), it seems that the scanning Auger spectroscope may soon become a viable research tool, and already it can be used to analyse the composition of a 10 Å-thick surface layer. In the scanning mode of operation, simultaneous composition analyses can be obtained from a large number of positions on the surface of a given specimen.

J. G. Wright and P. K. Leung (University of East Anglia) used in situ electron diffraction methods to observe thin films of amorphous $\mathrm{Co}, \mathrm{Fe}, \mathrm{Mn}$ and $\mathrm{Cr}$ prepared by evaporation under ultra-high vacuum onto a substrate at $4 \mathrm{~K}$. This work should provide final confirmation that amorphous metallic films are not caused simply by impurities in vacuum systems. In a similar vein, B. Cantor and R. W. Cahn (University of Sussex) described the structures of highly extended solid solutions in binary metal alloy films.

A large section of the conference was devoted to various electrical and magnetic properties of thin films. C. $H$. L. Goodman (Standard Telephone Laboratories, Harlow) pointed out that previous claims that chalcogenide and metal oxide glasses represent the biggest breakthrough since the transistor 